\title{
REVISÃO DE ASPECTOS TEÓRICOS E METODOLÓGICOS DO USO DE MAPAS COGNITIVOS NA ERGONOMIA
}

\section{REVISION OF THEORETICAL AND METODOLYCAL ASPECTS OF THE USE OF MAPS COGNITIVES IN THE ERGONOMICS}

\author{
Ângela Regina Poletto \\ Professora EAF/RS-SC e Doutoranda em \\ Ergonomia \\ Universidade Federal de Santa Catarina \\ Programa de Pós-Graduação em Engenharia de Produção e Sistemas \\ Rua Esteves Júnior, 574/303, Florianópolis - SC \\ 48 -33224237 - arpoletto@ hotmail.com \\ Ana Lúcia Ferraresi Schmitz \\ Pesquisadora INPEAU - CSE \\ Universidade Federal Santa Catarina \\ Rua dos Gansos, 59, Florianópolis - SC \\ 48 -32372363 - alf@ccs.ufsc.br \\ Leila Amaral Gontijo, Dra. \\ Programa Pós-Graduação em Engenharia de Produção e Sistemas \\ Universidade Federal de Santa Catarina \\ leila@deps.ufsc.br
}

\section{RESUMO}

Este estudo tem como objetivo realizar uma revisão dos aspectos teóricos e metodológicos das pesquisas sobre o uso de mapas cognitivos como um recurso de investigação na ergonomia. Com a finalidade de fundamentar a pesquisa exploratória, selecionar e identificar na literatura especializada, o Portal da Capes foi utilizado como ferramenta de busca através do Scopus, 
Scielo, Medline e Elsevier. Os mapas cognitivos constituem-se em um instrumento importante na observação do comportamento humano em condições específicas, destacando-se, por meio do seu estado da arte, como auxiliar em ergonomia na análise das atividades. Os mapas auxiliam o processamento de informações seletivas e apontam metas para a tomada de decisões nas atividades de trabalho.

Palavras-chaves: mapas cognitivos, instrumento de observação, atividade de trabalho, ergonomia.

\begin{abstract}
This study aims to review theoretical and methodological aspects of research carried out on the use of cognitive maps as an investigative resource in ergonomics. With the purpose of establishing the foundation of exploratory research, as well as selecting and identifying it in the specialized literature, the Portal Capes was the search engine used through Scopus, Scielo, Medline and Elsevier. Cognitive maps are an important instrument in the observation of human behavior under particular conditions. Their state of the art enhances their role in assisting ergonomics with activity analysis. The maps aid the processing of selective information and point to goals with regard to decision-making in work-related activities.
\end{abstract}

Keywords: cognitive maps, observation instrument, work-related activities, ergonomics.

\title{
1. INTRODUÇÃO
}

Os mapas cognitivos ou modelos mentais são utilizados na ergonomia como um recurso que tem a finalidade de auxiliar na solução de um problema, na tomada de decisões e na interpretação de novos eventos em atividades de trabalho.

A ergonomia, como uma disciplina orientada para uma abordagem sistêmica de todos os aspectos da atividade humana, necessita de uma abordagem ampla em seu campo de ação, nos aspectos físicos, cognitivos, sociais, organizacionais e ambientais. Neste cenário são sugeridos os mapas cognitivos como um instrumento auxiliar de reflexão que gera conhecimentos sobre o problema em questão.

Este estudo tem como objetivo realizar uma revisão dos aspectos teóricos e metodológicos das pesquisas em ergonomia que utilizaram os mapas cognitivos como uma ferramenta de investigação. Serão abordados os conceitos, as características, a classificação, a construção dos mapas cognitivos e o estado da arte das pesquisas.

Como artigo de revisão, o presente trabalho tem sua base principal em documentos disponíveis no Portal Capes. Os seguintes termos foram introduzidos: cognitive maps $e$ cognitive mapping. Utilizou-se Scopus, Scielo, Medline e Elsevier como base para a pesquisa 


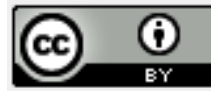

em periódicos, como: European Journal of Operacional Research, Technovation, British Journal of Management, e dissertações de mestrado e teses de doutorado. $\mathrm{O}$ artigo foi desenvolvido a partir destas referências na literatura.

\section{MAPAS COGNITIVOS}

\subsection{Conceito de Mapas Cognitivos}

Os estudos pioneiros de Ochanine (1966), na década de 70, contribuíram para as pesquisas sobre mapas cognitivos ao apresentar o conceito de imagem operatória como um esquema da situação real composto dos elementos mais importantes, o que permite a manipulação mental dessa situação, auxiliando o indivíduo no planejamento de ações e na resolução de um problema.

Pesquisadores utilizaram em suas pesquisas os mapas cognitivos como um recurso auxiliar no entendimento de problemas e situações na atividade de trabalho. A seguir serão apresentados conceitos que servem de base para o entendimento dos mapas cognitivos.

Bougon (1983), em seus estudos, definiu mapa cognitivo como um termo usado de forma genérica para representar possíveis padrões de relações entre conceitos. As palavras e frases que os indivíduos enunciam para expressar idéias ou conceitos em um dado contexto constituem os blocos para a construção do mapa cognitivo. No entanto, Cossette e Audet (1994) conceituam como "uma representação gráfica da representação mental que o pesquisador faz de um conjunto de representações discursivas enunciadas por um sujeito a partir de suas próprias representações cognitivas, a propósito de um objeto particular".

Na interpretação de Swan (1997), são representações, esquemas ou modelos mentais construídos pelos indivíduos, a partir das suas interações e aprendizagens em um domínio específico do seu ambiente, e que cumprem a função de dar sentido à realidade e permitemlhes lidar com os problemas e desafios que esta lhes apresenta. Na conceituação de Nicolini (1999), os mapas poderiam ser considerados como instrumentos de descrição e representação que ajudam na discussão e análise de alguns modos de pensamento e explicação dos eventos na cognição organizacional. 
Para Jardim (2006, p. 2), "essa representação gráfica é o resultado da interpretação mental que o analista (facilitador) faz a partir da representação discursiva feita pelo sujeito (ator) sobre um problema. Nesse processo discursivo-reflexivo-recursivo, representado pelo mapa cognitivo, preconiza-se a neutralidade por parte do facilitador".

Segundo Éden (1988), os mapas podem fornecer habilidade em escrever, estimular ou prognosticar o pensamento. Assim, as exigências que podem ser feitas para um mapa cognitivo como artefato é que eles podem representar dados subjetivos mais significativos do que outros modelos, propiciando subsídios para os pesquisadores interessados em conhecimento subjetivo, agindo como um instrumento que auxilie a tomada de decisão e negociação de problemas.

As definições apresentadas permitem afirmar que os mapas cognitivos são representações mentais auxiliares na análise, na discussão dos resultados, na negociação de problemas e nos desafios das atividades de trabalho.

\subsection{Características dos Mapas Cognitivos}

Os mapas cognitivos caracterizam-se por uma estrutura hierárquica, com maior freqüência na forma de significados/resultados, com o objetivo declarado no topo da hierarquia. No entanto, a forma hierárquica do mapa é informada freqüentemente por correntes circulares em que os significados e resultados dão laços em si mesmo. Nas pesquisas operacionais, a circularidade é considerada como uma estrutura fundamental, característica do mapa (EDEN, 1994).

Éden (2004) apresenta uma proposta na representação do mapa cognitivo. São desenhados em pequenos pedaços de texto, ligados com setas unidirecionais. Contudo, ressalta que a qualidade da representação depende da qualidade do entrevistador como ouvinte e intérprete. Os mapas não são apenas uma descrição gráfica do que é dito, antes eles são interpretações que são significadas pelo entrevistador.

Para o autor os mapas cognitivos têm propriedades que podem fornecer a visão de suas características gerais, sua complexidade está relacionada aos problemas/resultados como:

- A extensão do mapa - as análises sugerem que quanto mais conceito mais complexo será o mapa e o resultado: 
- A complexidade do mapa como uma rede - a robustez das análises depende da habilidade de codificação do mapeador. A inexperiência tende a gerar um mapa com poucos conceitos enquanto que a análise identificada pela experiência do mapeador gera mais setas;

- A idealização do pensamento - através dos mapas é possível representar o pensamento de alguém em situações no simples contexto de um sistema hierárquico, onde cada valor significa que o resultado é um simples valor ordenado.

O autor procura dar uma visão hierárquica ao mapa, considerando-o de fundamental importância para a sua estrutura. No entanto, Bastos (2002), em seu estudo, dá uma visão da esquematização dos mapas cognitivos apresentando características necessárias para a sua compreensão, tais como:

- Os mapas são representações dinâmicas do ambiente e devem ser atualizados a partir das experiências do sujeito;

- Os mapas não consistem em uma cópia do ambiente, mas uma representação ou modelo simplificado da realidade que fornece uma imagem aproximada (Laszlo et al., 1995). Esse processo é impreciso, não só porque a realidade está sempre em mudança, mas também pela natureza inferencial dos mecanismos envolvidos neste processo;

- Os mapas são flexíveis (podendo ser atualizados a todo o momento) e são utilizados para perceber as relações entre comportamentos variados e resultados semelhantes;

- O processo de esquematização do mapa cognitivo se dá pelo acesso do pesquisador ao material verbal;

- A esquematização e os elementos argumentativos são parciais, pois os indivíduos revelam aquilo que é útil aos seus objetivos e simplificam a realidade em função dos seus limites cognitivos. Nesse sentido, esse discurso é um produto semiótico e contextual e não consiste na expressão de uma realidade objetiva; no entanto, organiza as suas representações de acordo com as regras da lógica natural, possuindo um caráter persuasivo e pragmático.

Para os autores, os mapas cognitivos apresentam características peculiares em cada situação investigada em que a presença do mapeador exerce influência na construção e nos resultados obtidos, suas idéias são complementares na caracterização do mapa. 


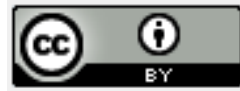

\subsection{Classificação dos Mapas Cognitivos}

A classificação dos mapas cognitivos que vem sendo mais utilizada é a de Huff (1990) e Fiol \& Huff (1992). Os autores classificaram os mapas quanto: ao tipo de mapa, ao tipo de análise, ao tipo de intervenção, ao uso e aos componentes.

Quanto ao tipo de mapa, podem ser de pontos e de contexto. Os mapas de pontos definem uma seqüência clara de pontos de escolha. Ele pode assumir uma forma gráfica, mas é facilmente memorizável e transferível de um indivíduo para o outro, verbalmente. Fazendo uma analogia com um mapa cartográfico de uma cidade, ele seria daquele tipo "saindo de um ponto $\mathrm{X}$, virar à direita, caminhar duas quadras, virar à esquerda e caminhar três quadras encontrando o ponto Y desejado". Os pontos de escolha são claros e, se não há incertezas sobre a rota ou problemas de obstáculos, ele evita distrações favorecendo a eficiência comportamental (Montibeller Neto,1996).

Os mapas de contexto contêm além dos pontos específicos de escolha, também a informação sobre o contexto envolvendo tais pontos. Estes mapas fornecem um senso de ambiente em que as decisões são tomadas e ainda permitem julgamento para busca de opções. Retornando à analogia cartográfica, eles fornecem detalhes sobre as características do terreno e sobre as formas com que tais características podem ser ligadas. Caso existam incertezas em uma seqüência de passos, os mapas de contextos permitem exercer o julgamento se for impedida uma dada seqüência ao longo do caminho. Por outro lado, a excessiva riqueza de detalhes pode distrair a ação do indivíduo que utiliza o mapa. Devido à sua complexidade ele requer uma representação gráfica além da oral (Montibeller Neto,1996).

Quanto ao tipo de análise, os mapas podem ser de análise hierárquica e cibernética. $\mathrm{Na}$ análise hierárquica a ênfase é na hierarquia dos componentes sob forma de uma racionalidade estratégica. $\mathrm{Na}$ análise cibernética a ênfase se dá nas características hierárquicas e os laços existentes entre os nós do mapa que levam às mudanças e ao crescimento estratégico.

Quanto ao tipo de intervenção, podem ser organizacionais ou individuais. Nos mapas organizacionais o facilitador procura um mapa coletivo que represente um instrumento para a ação da organização, seja como ferramenta de apoio à decisão ou para uma análise da 
organização. Os mapas individuais podem ter caracterização isolada, mas principalmente, podem ser usados para a obtenção dos mapas coletivos (organizacionais).

Quanto ao uso, os mapas cognitivos podem ser utilizados como produtos (mantendose estáveis no tempo) ou como ferramentas, com caráter dinâmico e passível de modificação (ou de abandono) por parte dos decisores, no enfrentamento de questões complexas.

Quanto aos componentes, podem ser de identidade, de categorização e causais. Os mapas de identidade são os que permitem descrever o "terreno cognitivo" ao identificarem os conceitos que as pessoas recuperaram para estruturar a sua compreensão de um problema ou domínio particular. Os mapas de categorização descrevem como os indivíduos organizam ou estruturam o seu conhecimento e, portanto, usam o processo de categorização apoiando-se nos seguintes pressupostos: o pensamento requer um resgate da memória organizada; o processo de categorização está envolvido na aprendizagem (modificação das velhas categorias e criação das novas); e o significado de qualquer conceito emerge no contraste com outros conceitos. Os mapas causais são os mais usados, sendo muitas vezes tomados como sinônimos de mapas cognitivos (LAUKKANEN, 1998). Em parte, pela primazia em compreender as condições que geram decisões de sujeitos singulares, face à necessidade de compartilhamento de visões e coordenação de cursos de ações. Essa necessidade, central na constituição do fenômeno organizacional, revela-se básica para a análise das práticas gerenciais e fundamentais para o êxito de qualquer empreendimento coletivo. Os mapas causais fornecem uma compreensão dos vínculos que os indivíduos estabelecem entre ações e resultados ao longo do tempo, assim como os pressupostos subjacentes aos julgamentos, de que uma ação levará a um resultado esperado.

As diferentes classificações dos mapas cognitivos permitem auxiliar os mapeadores na sua construção, facilitando os procedimentos de obtenção do conhecimento e o desenvolvimento da pesquisa.

\subsection{Construção dos Mapas Cognitivos}

Não há uma maneira única de se desenvolver um mapa cognitivo, mas diretrizes que auxiliam o mapeador nos aspectos gerais do mapeamento (RIEG E ARAÚJO FILHO, 2003). Para a construção de um mapa cognitivo são necessários alguns procedimentos de obtenção do conhecimento. É possível usar pronunciamentos, representados, via de regra, por 
codificações padronizadas, questionários ou entrevistas. As entrevistas podem ser nãoestruturadas, em que o pesquisador tem em mente apenas algumas regras de construção do mapa, desenvolvendo-o a partir do discurso do tomador de decisão. Existem também os mapas desenvolvidos a partir de entrevistas semi-estruturadas, que parecem melhor se adaptar à construção dos mapas quando da aplicação na AET - Análise Ergonômica do Trabalho (MONTIBELLER NETO, 2000).

A construção de um mapa cognitivo depende de dois fatores: a abordagem empática inicial, por parte do facilitador (mapeador), e o estabelecimento de um eficiente e legítimo processo de negociação. A aparente confusão nos primeiros mapas cognitivos faz parte do processo. A busca da significação e clareza deve ser alcançada pela prática. Objetivamente, o mapa cognitivo é uma hierarquia de conceitos relacionados por ligações meio e fim, que representa o sistema de valores do(s) decisor(es) na forma de objetivos estratégicos. O mapa cognitivo também fornece alternativas ou ações para atingir os objetivos estratégicos, através dos conceitos subordinados na hierarquia. Os autores ainda sugerem que por ser um processo que exige esforço mental e que pode tornar-se improdutivo devido ao cansaço, cada entrevista deve durar entre 60 e 90 minutos e ser realizada no ambiente do entrevistado ou em local neutro. Dessa forma, o entrevistado sente-se engajado na construção do mapa e o mapeador tê-lo-á como aliado no processo (EDEN e ACKERMAN, 1998).

Montibeller Neto (1996) aponta alguns passos para a construção dos mapas cognitivos:

- O primeiro passo é definir junto aos decisores um nome que descreva o problema que o facilitador (mapeador) irá apoiar na resolução. O facilitador deve inicialmente buscar escutar o que os decisores têm a dizer sobre seu problema. Nesse sentido, Rieg e Araújo Filho (2003) enfatizam a importância das entrevistas, para os autores nessa etapa o facilitador deve evitar interferir no que eles dizem (abordagem empática), pois isso poderia direcionar o nome de forma inadequada;

- O segundo passo é a realização de um brainstorming ${ }^{1}$ com o decisor à identificação dos Elementos Primários de Avaliação (EPAs) do mapa, que permitirão o início da construção do mapa. Esses elementos são constituídos de objetivos, metas, valores dos

3 Brainstorming (ou "tempestade de idéias") mais que uma técnica de dinâmica de grupo é uma atividade desenvolvida para explorar a potencialidade criativa do indivíduo, colocando-a a serviço de seus objetivos. Disponível em < http://en.wikipedia.org/wiki/Brainstorming, $>$. Acesso em 24/09/07. 
decisores, bem como de ações e alternativas de ação. O procedimento para obter os EPAs consiste em encorajar a criatividade, estimulando os decisores a expressar todos os elementos que vêm à mente, portanto, quanto mais EPAs mais rico poderá ser o mapa. O facilitador constrói os mapas a partir do que escuta e das declarações sobre porque certos eventos ocorreram. Assim, os EPAs são as anotações obtidas nas entrevistas e observações.

- A partir de cada EPA deve ser construído um conceito. Inicialmente o elemento primário de avaliação é orientado à ação, fornecendo assim o primeiro pólo do conceito. O sentido do conceito está baseado em parte na ação que ele sugere, portanto, o mapa deve ter uma perspectiva orientada à ação (ACKERMAN et al. 1991 apud MONTIBELLER NETO, 1996).

- Outra recomendação é questionar o decisor pelo pólo oposto psicológico. O pólo oposto é importante na medida em que o conceito só tem sentido quando existe o contraste entre dois pólos. Mas não seria o oposto lógico (contrário), mas ao significado aceito pelo ator como oposto ao EPA. Em cada pólo oposto os conceitos são separados por aspas. Os blocos devem ser pequenos para facilitar a compreensão do seu significado e a apresentação gráfica do mapa.

- Por fim, passa-se à construção da hierarquia dos conceitos, pode-se questionar o decisor sobre quais são os meios necessários para atingi-los, ou ainda, sobre quais são os fins os quais ele se destina.

Bastos (2002) apresenta as características metodológicas de construção dos mapas cognitivos, baseadas na classificação de Huff (1990) e Fiol e Huff (1992):

- Nos mapas de categorização são selecionados os elementos a serem mapeados, por considerações teóricas ou dos sujeitos. O sujeito classifica, hierarquiza e compara construtos julgados relevantes. A composição da matriz de dados é feita considerando a importância, a desejabilidade e a similaridade percebida.

- Nos mapas de identidade, os dados são coletados por entrevista semi-estruturada ou aberta. Envolve a criação de categorias e sua contagem por programa computadorizado. A partir da freqüência da evocação é possível construir gráficos que identificam as categorias mais citadas. 
- Os mapas causais utilizam três principais técnicas: Self-Q (técnica do autoquestionamento), CCM (Comparative Casual Mapping) - análise comparativa e SODA (Strategic Options Development Analysis) - que possuem caráter de metodologia de solução de problemas organizacionais.

Os mapas cognitivos compreendem internamente conceitos representados e a interrelação entre os conceitos que um indivíduo pode usar para entender eventos passados e interpretar novos eventos. Os tomadores de decisões têm limitado as capacidades para processar informações, enquanto que, tratando de problemas complexos, eles raramente poderiam processar todas as informações relevantes. Ajudam os tomadores de decisões a processar informações seletivamente e decidir o curso apropriado das ações (WEIK, 1979).

Como se pode observar, o mapeador tem influência fundamental na condução da construção dos mapas cognitivos, uma vez que não existe uma maneira padronizada, mas sim diretrizes que o orientam.

\subsection{Estado da Arte das Pesquisas sobre Mapas Cognitivos na Ergonomia Brasileira}

No Brasil, as pesquisas na área de ergonomia e ergonomia cognitiva têm utilizado os mapas cognitivos na análise das atividades como um instrumento que auxilia na representação de estruturas e processos cognitivos. A utilização de mapas cognitivos na ergonomia pode ser verificada através dos seguintes estudos brasileiros analisados.

O método da Análise Ergonômica do Trabalho com a aplicação dos mapas cognitivos foi utilizado no estudo desenvolvido por Franco (2001) em relação às estratégias desenvolvidas na gestão do conhecimento na construção civil, para tratar e esquematizar as informações obtidas na pesquisa de campo, realizada no estudo de caso (multicasos), referente aos gerentes do canteiro de obras de duas empresas construtoras. Os mapas serviram como instrumento de apoio à estruturação do conhecimento, visando à concepção ergonômica da tarefa. A partir das análises foi possível apresentar as exigências cognitivas e descrever a tarefa de estrutura em concreto. Os resultados mostram que para inovar o sistema construtivo é preciso não somente novas formas de gerenciar o processo, mas também gerenciar os conhecimentos e as exigências cognitivas para a realização do trabalho. A utilização de mapas cognitivos pode ser exemplificada neste estudo, conforme Figura 1. 


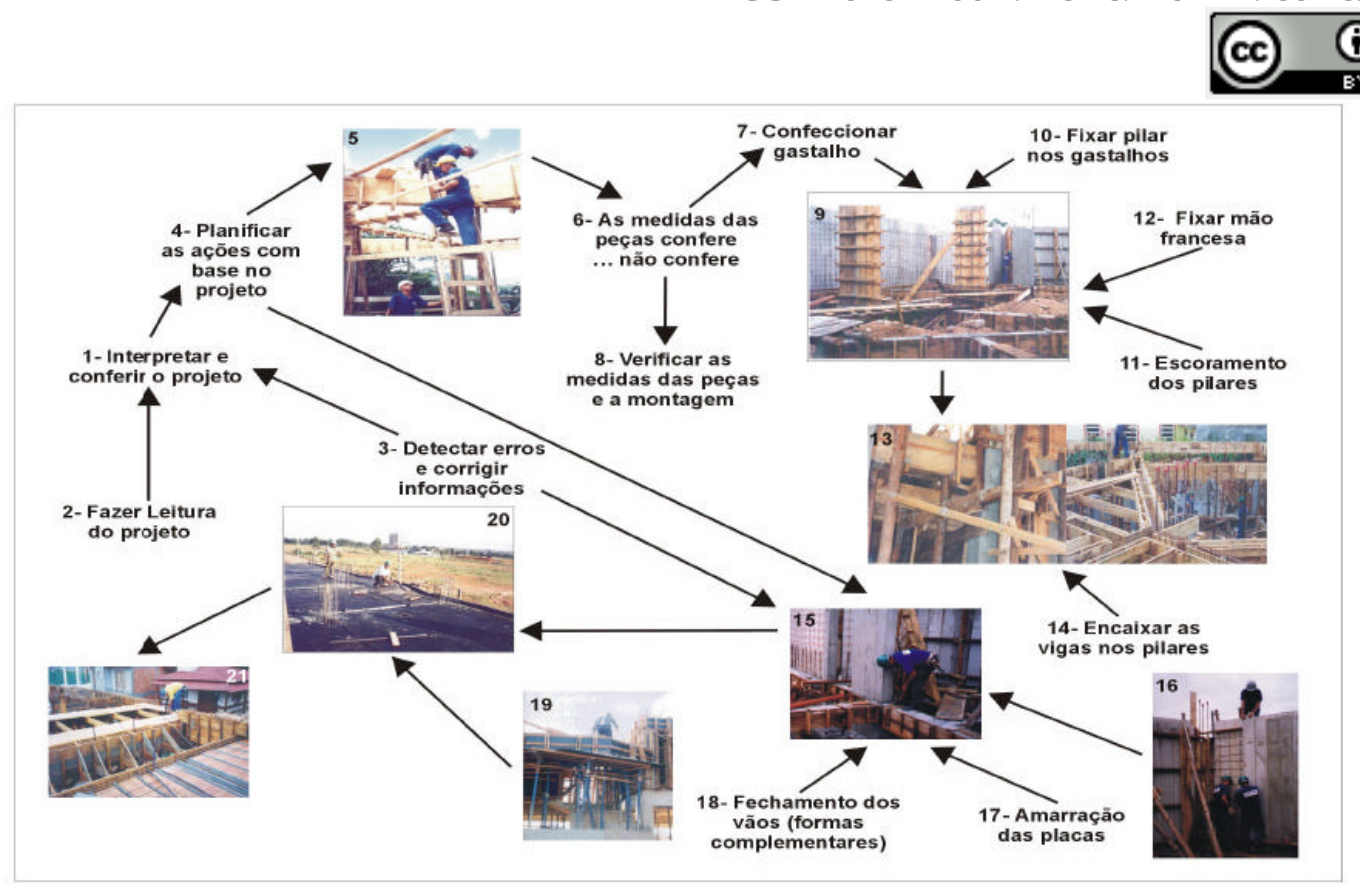

Figura 1: Mapa Cognitivo - Representação da Execução das Formas - Ilustrado Fonte: Franco (2001).

Dagostin (2003), em estudo de caso, em uma empresa do setor de caloríficos cerâmicos do município de Criciúma, SC, analisou as atividades de duas funções (ajudantes de produção e líderes de produção), no setor de fornos de fusão. Os mapas cognitivos foram apresentados como uma estratégia para a formulação de programas de capacitação, fundamentados no fato de que o mapeamento cognitivo fornece subsídios que permitem ao sujeito refletir sobre a sua própria visão e adquirir, com mais eficácia, conhecimentos sobre um determinado domínio. Através desse estudo foi concluído que a maioria dos programas de capacitação não leva em conta as reais atividades desenvolvidas, baseando-se nas tarefas prescritas, correndo o risco de se tornarem ineficazes para a realização do trabalho.

Os mapas cognitivos foram utilizados no estudo de Santos (2001) sobre a habitação popular concebida com o uso de malhas poliédricas não ortogonais, características pouco convencionais a uma edificação residencial. Sua base teórica encontra-se apoiada nos princípios da psicologia ambiental, da ergonomia e da ergonomia cognitiva, fundamentando os estudos sobre os conflitos originados do ato de habitar uma edificação, nas questões de ordem cognitivas. A pesquisa foi realizada com cinco pessoas durante a aquisição de uma nova casa, fazendo uso de recursos de simulação em realidade virtual proporcionando um melhor entendimento do projeto. Os resultados demonstraram que todos os atores envolvidos têm a necessidade de espaço ao ar livre. 


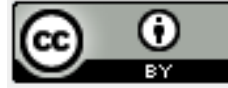

Assim, o sucesso ou insucesso de um projeto para construção de espaços construídos é de ordem subjetiva, em que a representação mental do usuário determina seus desejos e sentimentos em relação a esses espaços. A utilização dos mapas cognitivos permitiu a análise da adequação de projetos de arquitetura, promovendo uma complementaridade entre as ferramentas e demonstrando um melhor entendimento dos aspectos cognitivos e perceptivos envolvidos na relação entre o homem e o espaço construído que ocupa.

Brondani (2006), em sua tese sobre a percepção da luz artificial, no interior de ambientes edificados, avaliou como o usuário percebe a luz no interior de ambientes edificados. O tema foi tratado a partir de princípios e fundamentos da cor, dos canais sensoriais, da fisiologia humana, da luz e das atividades mentais e suas representações. Os mapas cognitivos foram utilizados como ferramenta para coleta de dados auxiliando nas análises dos desenhos onde foram identificados os elementos que influenciaram na percepção da luz. Os resultados demonstraram que a utilização correta da luz possibilita novas alternativas para diferenciar os ambientes habitáveis.

Os estudos demonstram que as pesquisas sobre os mapas cognitivos são um recurso viável para o melhor entendimento dos aspectos cognitivos na ergonomia, principalmente na análise das atividades. Esta abordagem de estudo dos mapas cognitivos na ergonomia tem sido verificada principalmente nas pesquisas brasileiras, em outros países tem sido utilizada com ênfase maior em estudos organizacionais.

\section{CONSIDERAÇÕES FINAIS}

Os mapas cognitivos constituem-se em uma maneira versátil e eficiente de complementar estudos quantitativos e também auxiliar em estudos qualitativos, em que as principais ferramentas para a coleta das informações são observações, entrevistas, questionários e medições (levantamento físico). Os dois primeiros associando-se especialmente a aspectos qualitativos, enquanto que os dois últimos estão relacionados mais aos quantitativos.

Os mapas cognitivos são utilizados como uma forma de comunicação da natureza de um problema, indicando a maneira como uma pessoa interpreta uma determinada situação. Portanto, auxiliam o processamento de informações seletivas e apontam metas para a tomada de decisões nas atividades de trabalho. 
Na ergonomia os mapas cognitivos servem como um recurso que utiliza os processos cognitivos na análise da atividade de trabalho e como uma ferramenta para investigar o comportamento humano frente a problemas específicos, fazendo inferências e auxiliando na análise ergonômica do trabalho.

\section{REFERÊNCIAS}

BASTOS, A. V. B. Mapas Cognitivos e a Pesquisa Organizacional: Explorando Aspectos Metodológicos. Estudos de Psicologia. Natal, v. 7, n. Especial, p. 65-77, 2002.

BOUGON, M. G. Uncovering Cognitive Maps: The Self-Q Technique. In: Morgan, G. (Org.). Beyond Method. Newbury Park: Sage, 1983.

BOUGON, M. G. Congregate cognitive maps: a unified dynamic theory of organization and strategy. Journal of Management, v. 29, n. 3, p. 369-389, 1992.

BRONDANI, S. A. A Percepção Da Luz Artificial No Interior De Ambientes Edificados. Florianópolis, 2006. Tese (Doutorado em Engenharia de Produção) - Programa de Pós Graduação em Engenharia de Produção, Universidade Federal de Santa Catarina.

COSSETTE, P.; AUDET, M. Qu'est-ce Qu'une Carte Cognitive? In: COSSETTE, P. (Org.), Cartes Cognitives et Organisations. Quebec: Les Presses de l'Únivesité Laval et les Éditions, ESKA, 1994.

DAgOstin, R. Mapas Cognitivos Como Suporte Para Programas de Capacitação: Um Estudo De Caso Com Base Na Análise Ergonômica Das Atividades. Florianópolis, 2003. Tese (Doutorado em Engenharia de Produção) - Programa de Pós Graduação em Engenharia de Produção, Universidade Federal de Santa Catarina.

EDEN, C. Cognitive Mapping. European Journal of Operational Research, n.36, p. 1-13, 1988. 
EDEN, C. Cognitive Mapping and Problem Structuring for System Dynamics Model Building. System Dynamics Review, n. 10, p. 257-276, 1994.

EDEN, C. Analyzing Cognitive Maps to Help Structure Issues or Problems. European Journal of Operational Research, n. 159, p. 673-686, 2004.

FRANCO, E. M. Gestão do Conhecimento na Construção Civil: Uma Aplicação dos Mapas Cognitivos na Concepção Ergonômica da Tarefa de Gerenciamento dos Canteiros de Obras. Florianópolis, 2001. Tese (Doutorado em Engenharia de Produção) Programa de Pós Graduação em Engenharia de Produção, Universidade Federal de Santa Catarina.

FIOL, C. M.; HUFF, A. S. Maps for Managers: Where Are We? Where do We Go from Here? Journal of Management Studies, v. 29, n. 3, p. 267-286, 1992.

HUFF, A. S. Mapping Strategic Thought. Chichester: Wiley \& Sons, New YorK, 1990.

JARDIM, S. B. Mapas Cognitivos: Um caminho para construir estratégias. Disponível em < http: www.pucrs.br/feng/dec/civil/professores/jardim/artigos-mapas-cognitivos.pdf $>$. Acesso em 27/07/2006.

LASZlO, E.; MASUlli, I.; ARTIGIANI, R.; CSÁNYI, V. The Evolution of Cognitive Maps - new Paradigms for the Twenty-first Century. Amsterdam: Gordon and Breach, 1995.

LAUKKANEN, M. Conducting Casual Mapping Research: Opportunities and Challenges. In: EDEN, C.; SPENDER, J. C. Managerial and Organizational Cognition: Theory, Methods and Research. London: Age Publications, 1998.

MONTIBEller NeTO, G. Mapas Cognitivos: Uma Ferramenta de Apoio à Estruturação de Problemas. Florianópolis, 1996. Dissertação Mestrado em Engenharia de Produção) - Programa de Pós Graduação em Engenharia de Produção, Universidade Federal de Santa Catarina. 
MONTIBEller NetO, G. Mapas Cognitivos Difusos para Apoio a Decisão: Uma Metodologia Integrada para Construção de Problemas e Exploração do Impacto de Alternativas nos Valores do Tomador de Decisão, Florianópolis, 2000. Tese (Doutorado em Engenharia de Produção) - Programa de Pós Graduação em Engenharia de Produção, Universidade Federal de Santa Catarina.

NICOLINI, D. (1999). Comparing Methods for Mapping Organizational Cognition. Organization Studies, n. 20, v. 5, p. 833-860, 1999.

OCHANINE, D. The operative image of controlled object in "Man-Automatic Machine" systems. In: XVIII CONGRESSO INTERNACIONAL DE PSICOLOGIA, 1966, Moscou. Anais... Moscou, 1966, p.48-56.

RIEG, D. L.; RAÚJO FILHO, T. Mapas Cognitivos Como Ferramenta e Resolução de Problemas: O caso da Pró-Reitoria de extensão da UFSCar. Gestão e Produção, n. 2, p. 145162, ago, 2003.

SANTOS. V. Modelo de Avaliação de Projetos - Enfoque Cognitivo e Ergonômico. Florianópolis, 2001. Tese (Doutorado em Engenharia de Produção) - Programa de Pós Graduação em Engenharia de Produção, Universidade Federal de Santa Catarina.

SWAN, J. Using Cognitive Mapping In Management Research: Decisions About Technical Innovation. British Journal of Management, n. 8, p. 183-198, 1997.

WEICK, K. E. The Social Psychology of Organizing. Addison-Wesley, Reading, MA, 1979.

WIKIPÉDIA. Brainstorming. Disponível em < http://en.wikipedia.org/wiki/Brainstorming, $>$. Acesso em 24/09/07. 